\title{
Support Vector Machines for Providing Selectivity of Distance Protection Backup Zone
}

\author{
A.L. Kulikov, D.I. Bezdushnyi *
}

\author{
Nizhny Novgorod State Technical University named after R.E. Alekseev, Nizhny Novgorod, Russia
}

\begin{abstract}
The development of present-day power systems is associated with the wide use of digital technologies and intelligent algorithms in control and protection systems. It opens up new opportunities to improve relay protection and automation hardware and develop its design principles. Simulation modeling becomes a new tool not only for studying power systems operation but also for designing new relay protection methods.

The use of simulation modeling in combination with machine learning algorithms makes it possible to create fundamentally new types of digital relay protections adaptable to a specific protected facility and able to use all the available current and voltage measurements to the fullest extent possible. Machine learning also allows developing auxiliary selective elements for improving the basic characteristics of existing relay protection algorithms such as selectivity, sensitivity, and speed of operation. The paper considers an example of designing an auxiliary element to provide selectivity in the backup zone of distance protection. The problem is solved using one of the most widely known machine learning techniques, i.e., the method of support vector machines (SVM).
\end{abstract}

Index Terms - multidimensional relay protection, support vector machines, distance relay

\section{INTRODUCTION}

Distance protection is one of the most widely spread and effective types of conventional non-unit relay protection. It is usually used in electrical grids with a complex configuration where the use of simpler overcurrent relays is unacceptable due to either their low sensitivity or inability to provide selectivity.Apart from the protection of its main zone, distance protection provides partial or

\footnotetext{
${ }^{*}$ Corresponding author.

E-mail: dimok.93@mail.ru
}

http://dx.doi.org/10.38028/esr.2020.02.0004

Received March 17, 2020. Revised May 02, 2020.

Accepted August 10, 2020. Available online October 21, 2020.

This is an open access article under a Creative Commons Attribution-NonCommercial 4.0 International License.

(C) 2020 ESI SB RAS and authors. All rights reserved. complete protection of the adjoining network components. This approach increases the reliability of relay protection systems and power supply systems in general.

It is worth noting that it is not always possible to provide effective fault detection in the distance protection backup zone with the non-unit protection scheme. Let us consider part of an electrical grid shown in Fig. 1. The second zone of distance protection of the power line $\omega_{1}$ must cover the entire line $\omega_{1}$ and partially back up protections of the parallel line $\omega_{2}$, the adjoining line $\omega_{3}$, and the transformer $\mathrm{T}_{1}$.

According to the regulations [1], the following restrictions are imposed on the value of reach settings of the second zone of distance protection (Table 1).

To evaluate the efficiency of the distance protection backup zone of line $\omega_{1}$, we will collect statistical data on faults on the components adjoining the given line. To this end, we will use the Monte-Carlo simulation method. The method suggests multiple simulations of the analyzed objects with a set of parameters randomly generated according to a given distribution law. In the model of the considered electrical grid, these parameters are rms value and angle of voltage in system A (ranges of values are given in Fig. 1, the distribution law is uniform), distance to the fault, and arc resistance. After all the parameters of the current experiment are obtained, a system of contour equations defining the equivalent circuit of the grid is built and solved. This allows determining the currents in branches and voltages at nodes based on which one can calculate the measurement of a distance protection impedance and other parameters of the operating conditions to be simulated.

Let us place a set of simulated short-circuit conditions in the adjoining components on a complex plane of distance protection of line $\omega_{1}$ and highlight those of them which make it impermissible for the second zone of distance protection of line $\omega_{1}$ to operate because of the offset conditions (Fig. 2).

Since the power line $\omega_{3}$ is relatively short (and hence has a low impedance), the condition of adjusting the reach settings $z_{\text {trip }_{\mathrm{w}_{1}}^{I I}}$ with $z_{\text {trip }_{\mathrm{w}_{3}}^{I}}^{I}$ according to (1) essentially limits the value of $z_{\text {trip }}^{I I}$.

Thus, a large part of faults on the parallel line $\omega_{2}$ and in the transformer $\mathrm{T}_{1}$ appear to be beyond the second zone of line $\omega_{1}$ (Fig. 2). Accurate calculations show that only 
Substation A

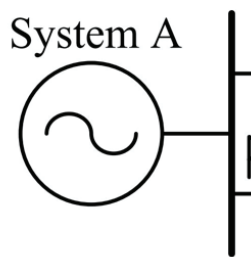

$\mathrm{U}=[216-219] \cdot \mathrm{e}^{-\mathrm{j} \cdot\left[9^{\circ}-10^{\circ}\right]} \mathrm{kV}$

$\mathrm{z}=6+120 \mathrm{j}$ Ohm

P - Relay Protection location

Substation B

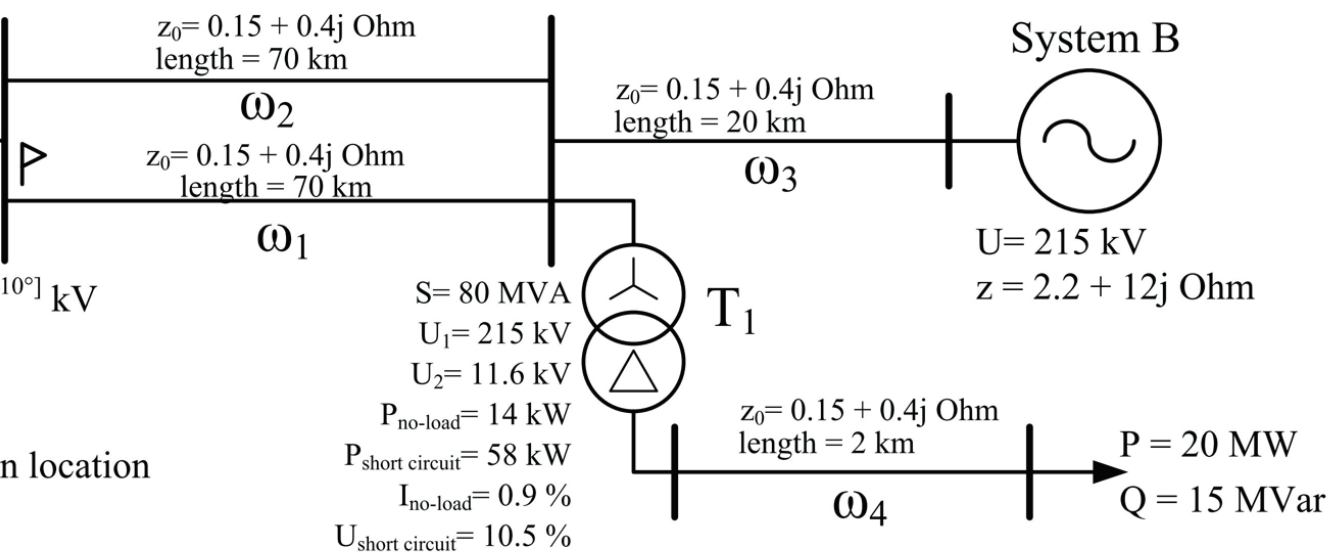

Fig. 1. The scheme of the analyzed electrical grid section.
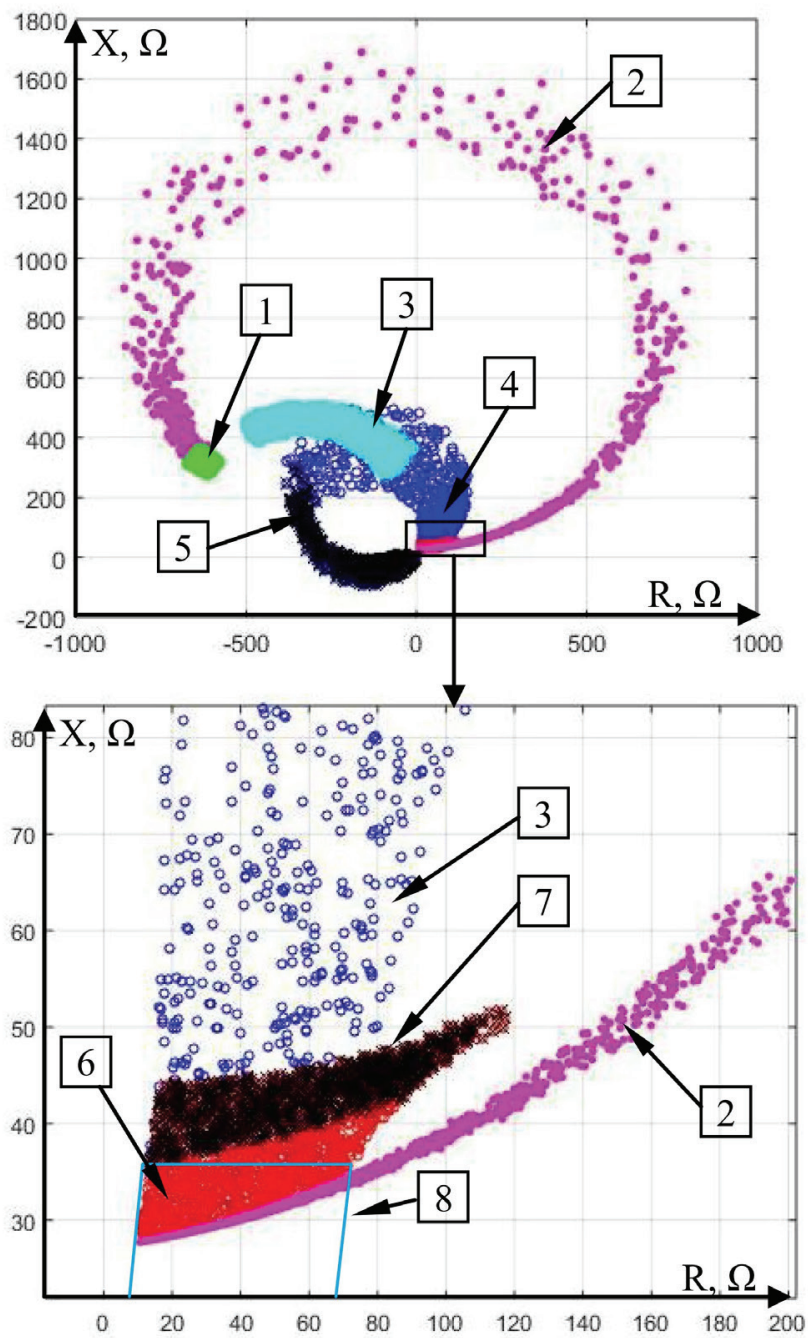

I - Normal conditions; 2 - Faults on $T_{\mathrm{I}}$ primary winding; 3 - Faults behind $T_{1}\left(\right.$ on $\left.\omega_{4}\right) ; 4$ - Faults on $\omega_{2} ; 5$ - Faults on $\omega_{2}$ (out of $\omega_{2}$ first zone); 6 - Faults on $\omega_{3} ; 7$ - Faults on $\omega_{3}$ (out of $\omega_{3}$ first zone); $8-\omega_{1}$ second zone characteristic;

Fig. 2. The results of the simulation modeling.
Table 1. Distance protection second zone reach setting conditions

\begin{tabular}{cl}
\hline \hline \multicolumn{1}{c}{ Expression } & \multicolumn{1}{c}{ Description } \\
$z_{\text {trip }_{\omega_{3}}}^{I I} \leq \frac{z_{\omega_{1}}+\frac{1-\alpha}{k_{\omega_{3}}} \cdot z_{\text {trip }}^{I} p_{\omega_{3}}}{1+\beta+\delta}$ & $\begin{array}{l}\text { Coordination with the first } \\
\text { zone of line } \omega_{3} \text { distance } \\
\text { protection; }\end{array}$ \\
$z_{\text {trip } \text { I }_{\omega_{1}}}^{I I} \leq \frac{z_{\omega_{1}}+\frac{1-\alpha}{k_{\omega_{2}}} \cdot z_{\text {trip }}^{I}}{1+\beta+\delta}$ & $\begin{array}{l}\text { Coordination with the first } \\
\text { zone of line } \omega_{2} \text { distance } \\
\text { protection at substation B; }\end{array}$ \\
$z_{\text {trip }}^{I I} \leq \frac{z_{\omega_{1}}+\frac{z_{T_{1}}}{k_{T_{1}}}}{1+\beta+\delta}$ & $\begin{array}{l}\text { Coordination with the faults } \\
\text { behind the transformer; }\end{array}$ \\
\hline \hline
\end{tabular}

$42.7 \%$ of faults in the primary winding of the transformer and $5.8 \%$ of faults on line $\omega_{2}$ fit this characteristic. It means that in the cases where the main protections fail, most of the faults on the parallel line and in the transformer will be cleared only by the third zone of distance protection of line $\omega_{1}$. Since the third zone has a longer operation time, the protected components are more likely to suffer severe damage caused by long-lasting overcurrent.

The improvement of distance protection and its reach zone expansion can be achieved by using special fault detection systems able to indicate a faulted component in the grid. With such systems, the second zone of distance protection of line $\omega_{1}$, which is set according to (1-3), can be replaced by three independent zones. These zones will have the same time settings, and their reach settings will meet one of the conditions (1-3), respectively. By combining the mentioned zones with the selection elements as shown in Fig. 3, the reach point of the distance protection can be shifted further in the backup zone (increase sensitivity), which will completely exclude the possibility of false operation.

Thus, to provide efficient backup protection of the electrical grid components with sufficient operation time it is appropriate to use special algorithms for identification of emergency states. The concept of multi-dimensional 


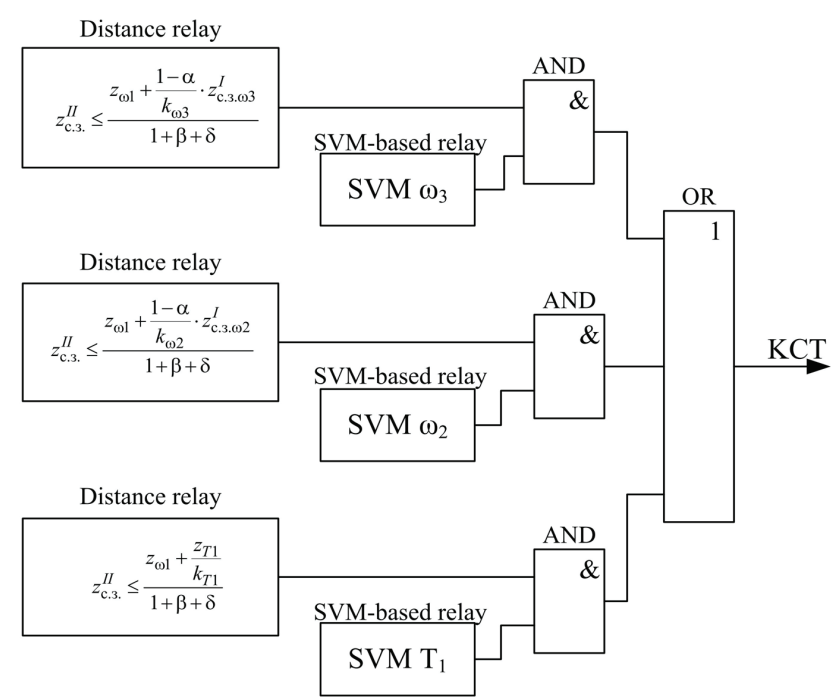

Fig. 3. The principle of combining the distance protection zones with the selection elements.

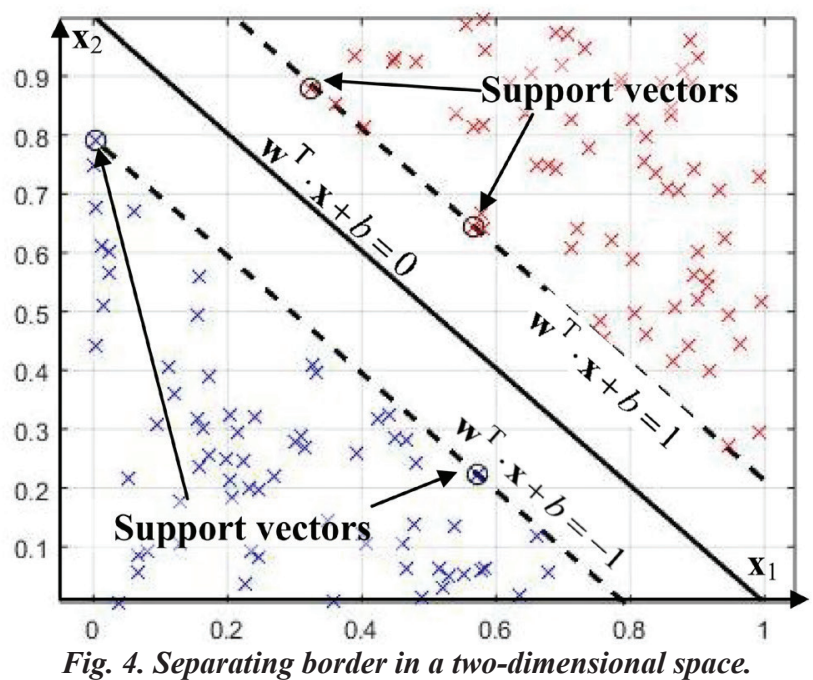

Table II $\alpha$ - and $\beta$-states for the designed elements.

\begin{tabular}{ccl}
\hline \hline Element & $\alpha$-states & \multicolumn{1}{c}{$\beta$-states } \\
\hline$S V M T_{1}$ & Faults on $T_{1}$ & $\begin{array}{l}\text { Faults behind } T_{1} ; \text { Faults on } \omega_{2} \text { outside } \\
\omega_{2} \text { zone } 1 ; \text { Faults on } \omega_{3} \text { outside } \omega_{3} \text { zone } \\
1 ; \text { Normal states. }\end{array}$ \\
$S V M \omega_{2}$ & Faults on $\omega_{2}$ & $\begin{array}{l}\text { Faults behind } T_{1} ; \text { Faults on } \omega_{3} \text { outside } \\
\omega_{3} \text { zone } 1 ; \text { Normal states. }\end{array}$ \\
$S V M \omega_{3}$ & Faults on $\omega_{3}$ & $\begin{array}{l}\text { Faults behind } T_{1} ; \text { Faults on } \omega_{2} \text { outside } \\
\omega_{2} \text { zone } 1 ; \text { Normal states. }\end{array}$ \\
\hline \hline
\end{tabular}

(multi-parameter) relay protection can be used to develop such algorithms.

This concept views relay protection as a discriminative system monitoring a specific set of information features, including operating parameters of the protected facility, which are available for measurement. The features are integrated into a feature space, and possible states of the protected facility (both feasible and unfeasible) constitute regions in the feature space similar to those on the impedance plane in Fig. 2. Unlike distance protection, however, the multi-dimensional approach uses features selected individually for a specific task, and their number (dimension of the feature space) can also vary. The features and their number should be selected based on the simplicity, illustrative representation, and sensitivity of the protection algorithm.

The decision rule of the multi-dimensional relay protection is usually obtained from an automated analysis of simulation data of the protected component. The procedure for classification of any observed state involves calculating coordinates of a point, which corresponds to a state in the selected feature space, and determining whether the obtained point is within the operation range set by the decision rule. In terms of machine learning, the implementation of multi-dimensional relay protection is a supervised classification problem, which, among other things, can be solved by the method of support vector machines.

The paper considers an example of using the support vector machines to design selective elements providing increased sensitivity of the second zone of distance protection.

\section{LITERATURE REVIEW}

The approach presented in [2-5] proposes a mapping of possible states of a protected facility onto one or several setting planes formed by arbitrary features. Similarly to machine learning methods, this approach assumes the protection decision rule to be formed via supervised classification. The simulation model of a protected component is usually used as a teacher. Based on the simulation results, the regions of normal and fault conditions are displayed on predefined setting planes. Unlike most of the machine learning techniques, this approach does not suggest the formation of regions in feature spaces with more than two dimensions.

There are also solutions providing transformer backup protection using both one-sided [6] and two-sided measurements [7] based on simulation data analysis. Like [2-5], the studies in [6] and [7] suggest dividing the $\mathrm{N}$-dimensional feature space into several setting planes. Also, the study discussed in [6] proposes a criterion for assessment of a feature space, which is based on the ratio of average Euclidean distance between the representatives of one class to the distance between representatives of different classes. The problem of feature space assessment was further developed in [8] where apart from the method that estimates the separability of states via metric functions, the authors describe the methods based on "separating functions" that explicitly define the border surface (linear or non-linear) between different classes in the feature space.

Different ways of fault detection in power systems based on machine learning methods are analyzed in various research papers. In [9], the authors compare the efficiency of classification algorithms such as Naïve Bayes' Classifier, 

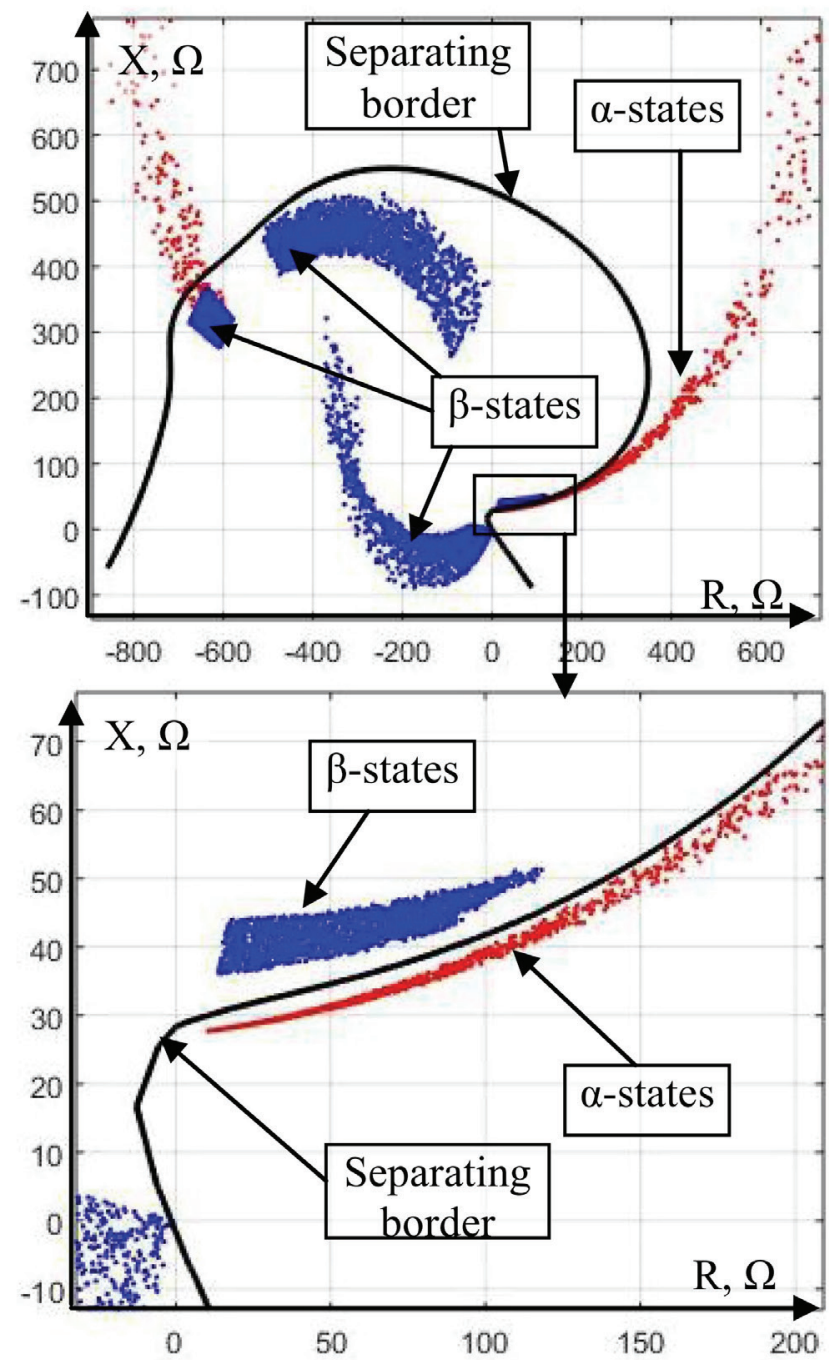

Fig. 5. Training set and separating border of the "SVM T1" element.

Radial Basis Function Classifier, Bagging and Boosting in the problem of identifying the type of a fault on a power line. In [10], the focus is on the relay protection based on the support vector machines in distributed networks with distributed generation. The authors used a feature space consisting of active and reactive power flows.

\section{THE APPLICATION OF SUPPORT VECTOR MACHINES}

In its simplest form, the support vector machine (SVM) technique is used for binary classification. The learning procedure assumes setting a hyperplane in the feature space to divide the training set elements related to different classes (denote the classes by $\alpha$ and $\beta$, respectively). Thus, the feature space is divided into an operation region and a non-operation region. The procedure for the state classification is to determine on which side of the hyperplane the considered state is.

The training set is said to be linearly separable if a hyperplane can be drawn so that all the points belonging to class $\alpha$ will lie on one side of it while all the points
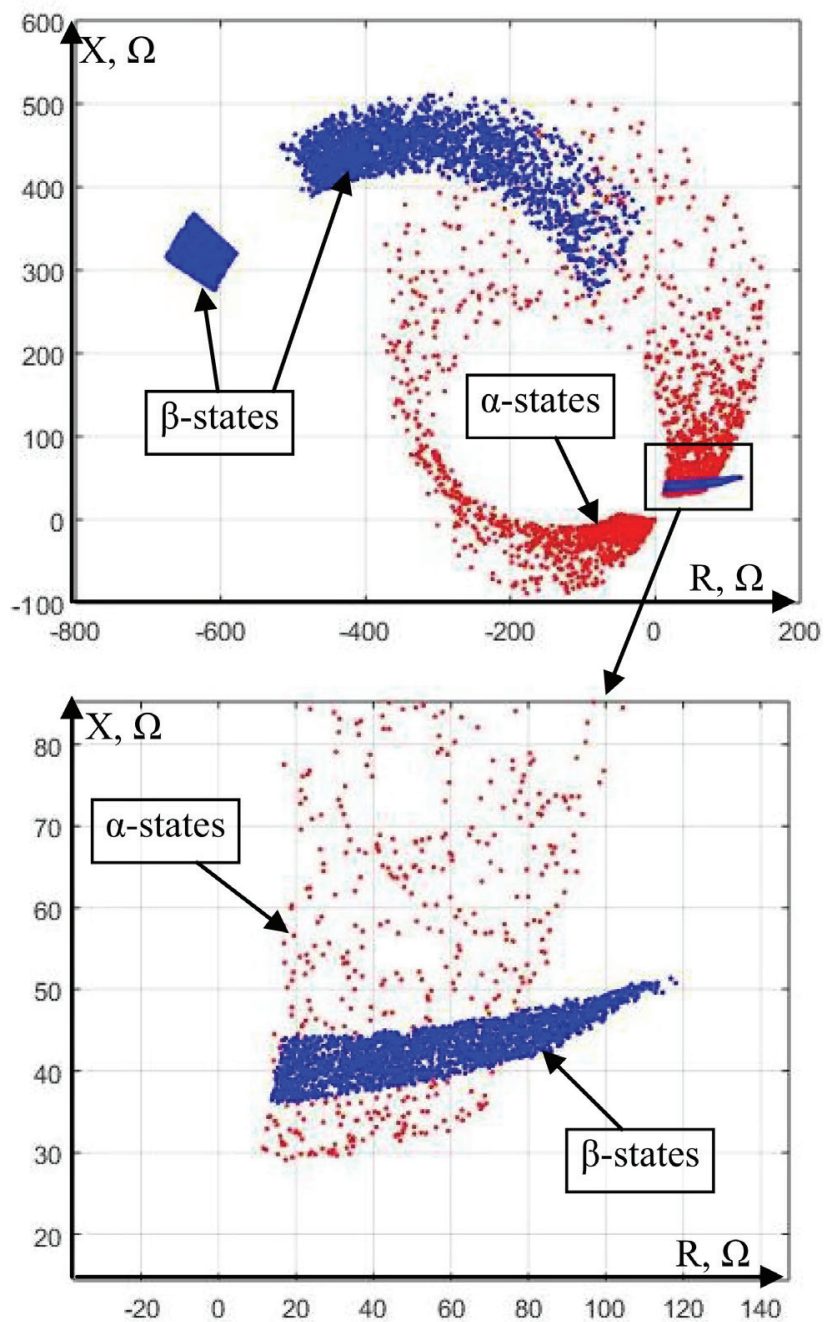

Fig. 6. Distribution of $\alpha$ - and $\beta$-states of the "SVM $\omega 2$ " element on the impedance plane.

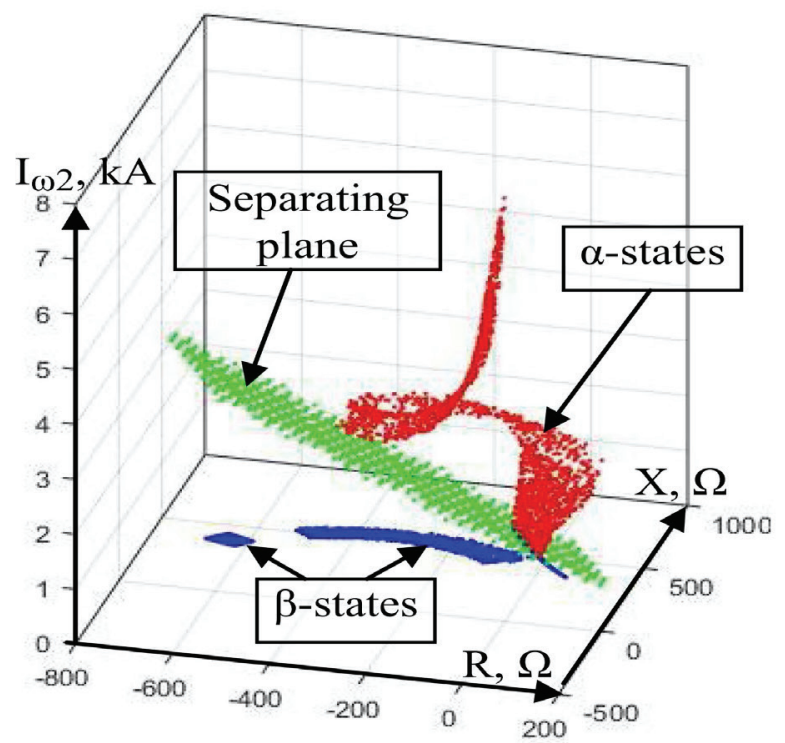

Fig. 7. Separating plane of the "SVM $\omega 2$ " element in a three dimensional space. 
belonging to class $\beta$ will be on the other side. This condition can be formalized as follows:

Let there be a training set $\mathbf{x}_{1}, \ldots, \mathbf{x}_{N}$ consisting of $N$ vectors in a multi-dimensional space. For each element of the training set there is a class tag $y_{1}, \ldots, y_{N}$ so that $y_{i}=$ 1 if the $i^{\text {th }}$ element belongs to the class $\alpha$ and $y_{i}=-1$ if the $i^{\text {th }}$ element belongs to the class $\beta$. Then the hyperplane $\mathbf{w}^{\mathrm{T}} \cdot \mathbf{x}+b=0$ separates the classes if the condition is met:

$$
\forall i \in 1, \ldots, N, y_{i} \cdot\left(\mathbf{w}^{\mathrm{T}} \cdot \mathbf{x}_{i}+b\right)>0 \text {. }
$$

An example of a separating hyperplane is shown in Fig. 4. Sum of distances between the plane and the closest representatives of both classes is called "margin". It is obvious that the most optimal hyperplane is the one that gives maximal margin provided that the condition (4) is met. It is proven [11] that equation of such a hyperplane can be obtained by finding the constrained minimum of the system of expressions:

$$
\left\{\begin{array}{c}
\min _{\mathbf{w}, b} \frac{1}{2} \mathbf{w}^{\mathrm{T}} \cdot \mathbf{w}, \\
\forall i \in 1, \ldots, N, \quad y_{i} \cdot\left(\mathbf{w}^{\mathrm{T}} \cdot \mathbf{x}_{i}+b\right) \geq 1 .
\end{array}\right.
$$

In most cases, however, the training set is not linearly separable, which means that there is no hyperplane satisfying the system of inequalities (4). In this case, the SVM allows misclassification of some elements from the training set but introduces a penalty for it. The optimization problem takes the following form:

$$
\begin{gathered}
\min _{\mathbf{w}, b, \xi_{1}, \ldots, \xi_{N}} \frac{1}{2} \mathbf{w}^{\mathrm{T}} \cdot \mathbf{w}+C \cdot \sum_{i=1}^{N} \xi_{i}, \\
\forall i \in 1, \ldots, N, y_{i} \cdot\left(\mathbf{w}^{\mathrm{T}} \cdot \mathbf{x}_{i}+b\right) \geq 1-\xi_{i}, \\
\forall i \in 1, \ldots, N, \xi_{i} \geq 0,
\end{gathered}
$$

where is a slack variable describing the classification error of the $i^{\text {th }}$ element of the training set; $\mathrm{C}$ is a coefficient defining penalty for the misclassification.

The coefficient $\mathrm{C}$ defines what will be more important for the classification algorithm: margin maximization or classification error minimization.

In practice, instead of the primal SVM problem (6), the dual form is solved, which provides the same answer but can be solved with faster numerical methods. Solving the dual SVM problem involves optimization of the variables $\lambda_{1}, \ldots, \lambda_{n}$ (expression (7)), i.e. Lagrange multipliers. The dual form is derived from the primal form [12] and is written as follows:

$$
\begin{gathered}
\min _{\lambda_{1}, \ldots, \lambda_{n}}\left(\sum_{i=1}^{n} \lambda_{i}-\frac{1}{2} \cdot\left(\sum_{i=1}^{n} \sum_{j=1}^{n} \lambda_{i} \lambda_{j} y_{i} y_{j} \mathbf{x}_{i}^{\mathrm{T}} \mathbf{x}_{j}\right)\right), \\
\forall i \in 1, \ldots, N, \quad 0 \leq \lambda_{i} \leq C \\
\forall i \in 1, \ldots, N, \quad \lambda_{i} y_{i}=0
\end{gathered}
$$

The quadratic programming problem (7) can be solved via one of the known methods [e.g. 13].
Having obtained optimal Lagrange multipliers $\lambda_{1}, \ldots$, $\lambda_{n}$ - by solving problem (7), one can restore separating hyperplane equation (8).

$$
\begin{aligned}
& \mathbf{w}=\sum_{i=1}^{n} \lambda_{i} y_{i} \mathbf{x}_{i}, \\
& b=\frac{1}{y_{S}}-\mathbf{w} \cdot \mathbf{x}_{S},
\end{aligned}
$$

where $s$ is an index of a vector such that $0<\lambda_{s}<C$.

The classification algorithm can be expressed in terms of the coefficients $\lambda_{1}, \ldots, \lambda_{n}$ as follows:

$$
a(\mathbf{x})=\operatorname{sign}\left(\sum_{i=1}^{n} \lambda_{i} y_{i} \mathbf{x}_{i}^{\mathrm{T}} \mathbf{x}-b\right) .
$$

Another way to classify the linearly inseparable data is to use a kernel trick. Its idea is to transform the original feature space $X$ to a higher dimensional space $H$ via a special kernel function $H=\varphi(X)$ in which the training set would be linearly separable. With such an approach, the optimization problem takes the form (10) and a new object is classified according to expression (11).

$$
\begin{gathered}
\min _{\lambda_{1}, \ldots, \lambda_{n}}\left(\sum_{i=1}^{n} \lambda_{i}-\frac{1}{2} \cdot\left(\sum_{i=1}^{n} \sum_{j=1}^{n} \lambda_{i} \lambda_{j} y_{i} y_{j} \varphi\left(\mathbf{x}_{i}, \mathbf{x}_{j}\right)\right)\right), \\
\forall i \in 1, \ldots, N, \quad 0 \leq \lambda_{i} \leq C, \\
\forall i \in 1, \ldots, N, \lambda_{i} y_{i}=0 . \\
a(\mathbf{x})=\operatorname{sign}\left(\sum_{i=1}^{n} \lambda_{i} y_{i} \varphi\left(\mathbf{x}_{i}, \mathbf{x}_{j}\right)\right) .
\end{gathered}
$$

The most widespread kernel functions used in practice are the polynomial (12) and the radial (13) ones.

$$
\begin{gathered}
\varphi\left(\mathbf{x}_{i}, \mathbf{x}_{j}\right)=\left(\mathbf{x}_{i} \cdot \mathbf{x}_{j}+1\right)^{d} \\
\varphi\left(\mathbf{x}_{i}, \mathbf{x}_{j}\right)=e^{-\gamma \cdot \mathbf{x}_{i}-\left.\mathbf{x}_{j}\right|^{2}}
\end{gathered}
$$

where $d$ is the degree of the polynomial; $\gamma$ is a regulation parameter.

It is worth noting that for the classification according to (9) it is sufficient to summate only those $i$, for which $\lambda_{i} \neq 0$, i.e. only the support vectors whose quantity is much smaller than the size of the whole training set.

\section{DESIGN OF THE SVM-BASED STATE CLASSIFIERS FOR RELAY PROTECTION}

The most important part of designing multi-parameter relay protection is the identification of states to be detected by the algorithm ( $\alpha$-states) and also the states ( $\beta$-states), at which the algorithm operation must be excluded. The composition and size of the training set will depend on this decision. Usually, $\alpha$-states are specific types of faults inside the reach zone, whereas $\beta$-states are operating conditions and faults outside the reach zone where tripping can lead to non-selective behavior of the protection. 
Using the above-described Monte-Carlo-based approach, we will form training and testing sets for the auxiliary elements to be designed.

The "SVM T," element (Fig. 3), which has to detect faults in the power transformer, should use simulation results of faults at $T_{1}$ as $\alpha$-states. The $\beta$-states should consist of the following scenarios:

- Faults behind $\mathrm{T}_{1}$;

- Faults on the part of line $\omega_{2}$ outside the reach of the distance protection first zone of line $\omega_{2}$;

- Faults on the part of line $\omega_{3}$ outside the reach of the distance protection first zone of line $\omega_{3}$;

- Normal states.

Note that the behavior of the "SVM $T_{1}$ " element in the situations that are not included in the training set is not pre-defined. For example, this element can operate in case of a fault at the beginning of line $\omega_{3}$, but this is permissible because it will not lead to non-selective switching off. Similarly, we can identify $\alpha$ - and $\beta$-states for the "SVM $\omega_{2}$ " and "SVM $\omega_{3}$ " elements (Table II).

Location of $\alpha$ - and $\beta$-states of the "SVM $T_{l}$ " element in the feature space formed by the impedance plane is shown in Fig. 5. An analysis of the Figure shows that in the given feature space, the regions of the states are linearly inseparable. Thus, the efficient classification via SVM is possible only by using a kernel function. Let us implement the procedure for learning the given training set with the aid of the polynomial kernel with a degree of 6 . The obtained separating border is also shown in Fig. 5.

The distance protection combined with the implemented "SVM $T_{1}$ " element detects $95 \%$ of phase-to-phase faults in the transformer primary winding and excludes only the faults that are close to the transformer neutral and practically do not differ from the normal state.

Location of $\alpha$ - and $\beta$-states of the "SVM $\omega_{2}$ " element in the same feature space is shown in Fig. 6.

As evidenced by analysis, Fig. 6, unlike Fig. 5, has overlays of $\alpha$ - and $\beta$-states, which makes it impossible to find a border that would accurately separate the studied and alternative states. One of the possible options to improve accuracy is to increase the dimension of the feature space. The rms value of the line $\omega_{2}$ current measured at the substation, where the designed protection should be installed, can be added to the feature space as the third dimension. In the resulting three-dimensional feature space, it becomes possible to place a separating plane providing unmistakable differentiation of the considered states (Fig. 7).

Figure 8 indicates a distribution of $\alpha$ - and $\beta$-states on the distance protection two-dimensional impedance plane for the " $S V M \omega_{3}$ " element designed to detect faults on line $\omega_{3}$. In terms of the classification, the case in Fig. 8 is the simplest because the accurate classification of states can be performed in the two-dimensional feature space without a specific kernel function. However, the use of a polynomial kernel function, for example, is preferable, since in this case, the separating border settles further from the complex values of both $\alpha$ - and $\beta$-states.

The use of a more complex kernel function guarantees a higher probability of appropriate work of the classifier in situations where the observed complex values go slightly beyond the regions formed by the simulation results.

Thus, the results presented in the paper allow us to conclude that the designed SVM-based auxiliary elements for distance protection identify the faulted components of the grid almost unmistakably and provide selectivity of the distance protection in the backup zone.

It is worth noting that the obtained separating borders in the feature spaces and their discriminative ability are characteristic only of a specific scheme and operating situation of a particular power grid. Therefore, the use of the SVM method in relay protection is limited despite its obvious benefits.

\section{CONCLUSION}

1. The advent of digital technologies and intelligent algorithms in the energy industry opens up new opportunities not only for the improvement of relay protection hardware but also for the development of its design principles. Simulation modeling becomes a powerful tool for the research into power system operation and the development of new relay protection algorithms.

2. The SVM application to relay protection problems is promising in both the formation of new protection algorithms and the use of SVM as an additional tool for increasing selectivity and speed of existing types of protection. An increase in the dimension of feature space to identify the electrical grid states significantly increases the probability of correct classification.

3. The proposed approach to the state recognition based on electrical grid simulation modeling and SVM allows the decision rule of protection algorithm to be adapted to an arbitrary grid configuration. It may be particularly appropriate for the grids of a complex configuration with the state parameters influenced by many factors (circuit breakers states, load power, and others), which cannot be handled manually.

4. Despite the high recognition ability of machine learning algorithms in general and the SVM in particular their application in relay protection is limited for several reasons:

- Trained algorithms are only applicable to a particular power grid section;

- Simulation modeling is required for setting the algorithm parameters;

- The use of a feature space with more than two dimensions makes it difficult to visualize the simulated states and the operation boundary obtained by implementing the algorithm. 


\section{REFERENCES}

[1] Guidelines for setting calculations of microprocessor relay protection and automation devices manufactured by «EKRA» RPE Ltd, «GE MULTILIN» and «ALSTOM GRID»/«AREVA» for one-way 110-330 $\mathrm{kV}$ overhead and cable lines. Organization standard, 56947007-29.120.70.200-2015, Apr. 2015.

[2] Y. Y Lyamets, G. S. Nudelman, D.V. Zinoviev, Multidimensional relay protection, part 1. Theoretical background, Electricity, no. 10, pp 17-25, 2009.

[3] Y. Y Lyamets, G. S. Nudelman, D.V. Zinoviev, Multidimensional relay protection, part 2. Analysis of recognition ability of a relay, Electricity, no. 11, pp 9-15, 2009.

[4] Y. Y Lyamets, G. S. Nudelman, I. S. Podshivalina, Y.V. Romanov, Multidimensionality effects in relay protection, Electricity, no. 9, pp 48-54, 2011.

[5] Y. Y Lyamets, P.I. Voronov, M.V. Martynov, Recognition ability of a power line's adaptive distance protection, Electricity, no.10, pp 13-19, 2015.

[6] I.V. Nagai, Providing backup function of transformer's relay protection in conditions of transverselongitudinal asymmetry, Izvestia of Universities. North Caucasus region. Technical sciences, no. 5, pp 19-24, 2011

[7] S.L. Kuzhekov et al., Power line differential phase protection with backup of relay protection of power transformers and circuit breakers connected to taps, Electrical Energy. Transmission and Distribution, no. 1, pp 108-115, 2019.

[8] I.V. Nagai, V.I. Nagai, S.V. Sarry, S. Yu. Berezkina, Estimation method of information features characterizing electrical grid modes by the criterion of recognizability by relay protection, Izvestia of Universities. North Caucasus region. Technical sciences, no. 4, pp 27-33, 2017.

[9] A.N. Hasan, P.S. Pouabe, B. Twala, The Use of Machine Learning Techniques to Classify Power Transmission Line Fault Types and Location, International Conference on Optimization of Electrical and Electronic Equipment, pp. 221-226, 2017.

[10] A X. Zheng, X. Geng, L. Xie, D. Duan, L. Yang and S. Cui, A SVM-based setting of protection relays in distribution systems, IEEE TPEC, 2018, pp. 1-6.

[11] L. Wang, Support Vector Machines: Theory and Applications, Berlin: Springer, 2006.

[12] C.M. Bishop, Pattern Recognition and Machine Learning, Berlin: Springer, 2006.

[13] L. Bottou and C-J. Lin, Support Vector Machine Solvers, Large Scale Kernel Machines, no.1, pp 1-28, 2007.

[14] A.L. Kulikov, D.I. Bezdushniy, The formation of generalized characteristics of the operation of relay protection based on the principal components analysis, Relay protection and automation, vol.34, no.1, pp. 20 27, 2019.
[15] A.L. Kulikov, D.I. Bezdushniy, M.V. Sharygin and V. Yu. Osokin, Analysis of the support vector machine implementation in the multi-dimensional relay protection, Izvestia RAN. Energetika, no. 2, pp 123$132,2020$.

[16] S. Abe, Support Vector Machines for Pattern Classification, Springer, 2010.

[17] N. Cristianini, J. Shawe-Taylor, An introduction to Support Vector Machines and other kernel-based learning method, Cambridge: Cambridge University Press, 2013.

[18] V. N. Vapnik, Statistical Learning Theory, New York, NY, USA: Wiley, 1998.

[19] K. Ravishankar and D. Thukaram, Support Vector Machine based High speed Protection Relay for EHV/ UHV Transmission Line. Presented at ICPCES.2012.

[20] K. Seethalekshmi, “A Classification Approach Using Support Vector Machines to Prevent Distance Relay Maloperation Under Power Swing and Voltage Instability", IEEE T Power Deliver, vol. 13, no. 27(3), pp. 1124-1133, July 2012. DOI: 10.1109/ TPWRD.2011.2174808.

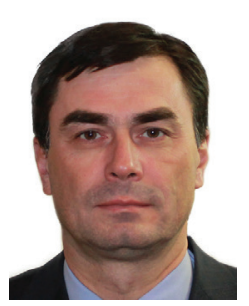

Alexander L. Kulikov, Ph.D., professor at the Department of Power Engineering, Power Supply and Power Electronics of Nizhny Novgorod State Technical University named after R.E. Alekseev. A.L. Kulikov graduated from Military Radio Engineering Academy of Air Defense named after V.I. Govorov L.A. in 1992. In 2007, he received his Ph.D. degree from Ivanovo State Power University named after V.I. Lenin. His research interests are relay protection, fault location, digital signal processing.

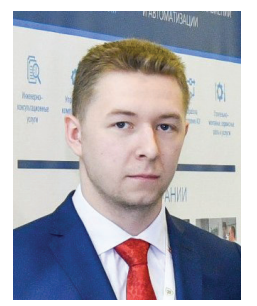

Dmitry I. Bezdushnyi, post-graduate at the Department of Power Engineering, Power Supply and Power Electronics of Nizhny Novgorod State Technical University named after R.E. Alekseev. D.I. Bezdushnyi received his Master's degree from Nizhny Novgorod State Technical University named after R.E. Alekseev in 2016. His research interests are relay protection, machine learning, simulation modeling. 